\title{
Diagrams in mathematics: history and philosophy
}

\author{
John Mumma • Marco Panza
}

Published online: 7 September 2011

(C) Springer Science+Business Media B.V. 2011

\section{Introduction}

Diagrams are ubiquitous in mathematics. From the most elementary class to the most advanced seminar, in both introductory textbooks and professional journals, diagrams are present, to introduce concepts, increase understanding, and prove results. They thus fulfill a variety of important roles in mathematical practice. Long overlooked by philosophers focused on foundational and ontological issues, these roles have come to receive attention in the past two decades, a trend in line with the growing philosophical interest in actual mathematical practice. Seminal contributions include the historical/philosophical analysis of diagrams in Euclid's geometry offered by Manders in his 1995 paper (2008), ${ }^{1}$ The date of publication of Manders' paper is misleading. Though it was not published until 2008, it was written in 1995 and widely circulated, and became highly influential as a manuscript. the logical studies of diagrammatic reasoning contained in Gerard Allwein and Barwise's compilation (1996), and Netz's historical study on the place of diagrams in Greek geometry (1999).

These works exhibit a broad range of intellectual perspectives. To bring people from these separate perspectives together, two workshops devoted to the history and philosophy of diagrams in mathematics were jointly organized by Departments of Philosophy and Classics at the University of Stanford, and REHSEIS (a research center

\footnotetext{
1 The date of publication of Manders' paper is misleading. Though it was not published until 2008, it was written in 1995 and widely circulated, and became highly influential as a manuscript.
}

\section{J. Mumma}

Division of Logic, Methodology, and Philosophy of Science at the Suppes Center for History and Philosophy of Science, Stanford University, Palo Alto, CA, USA

e-mail: john.mumma@gmail.com

M. Panza (ه)

CNRS, IHPST (UMR 8590 of the CNRS, the Univ. of Paris 1, and the ENS - Paris), Paris, France e-mail: marco.panza@univ-paris1.fr 
of the CNRS and the University of Paris 7). The first workshop was held at Stanford in the fall of 2007, the second in Paris in the fall of 2008. The present special issue is the direct result of these workshops.

Because of the central position of Euclid's geometry, in both the history and philosophy of mathematics, the diagrams of Euclid's geometry have been a major topic in the recent research on diagrams in mathematics. This is reflected in the make-up of the issue. Part I, which contains over half of the contributions to the issue, concerns Euclidean diagrams, addressing either their place and nature in the manuscript tradition, their role in Euclid's geometry, or their relevance to early modern philosophy and mathematics. The contributions of part II examine mathematical diagrams in more modern and/or advanced settings. In the remainder of this introduction, we briefly describe the content of these papers.

The two articles included in section I. $a$ are devoted to geometric diagrams in the manuscript tradition of ancient Greek texts. As Saito points out in the beginning of his article, the diagrams we find in modern versions of ancient Greek mathematical texts are far from representative of those appearing in the manuscripts from preceding centuries. These manuscripts result from various scribal traditions that exhibit various practices in the production of diagrams. The general aim of both Saito's article and Gregg De Young's is to illuminate significant features of such practices.

Saito's concern is with how the diagrams of the manuscript tradition relate to the geometrical content of the arguments they accompany. They often satisfy conditions not stipulated to hold (e.g. by instantiating a parallelogram as a square) or alternatively misrepresent conditions stipulated or proven to hold (e.g. by depicting angles stipulated as equal as clearly unequal). There are, further, notable features with the diagrams representing reductio ad absurdam arguments, and the diagrams representing arguments that range over many cases. To shed light on the different manuscript traditions in these respects, Saito presents a case study of the manuscript diagrams that accompany two propositions in book III of Euclid's Elements.

De Young focuses on a specific manuscript tradition. He explores the basic "architecture" (i.e. the relation of diagrams with white space and text) of medieval manuscripts and early printed editions of Euclidean geometry in the Arabic transmission. Many features of Euclidean diagrams remain constant through a long transmission history, although differences in scribal abilities exist. Yet there also seems to be a degree of freedom to adapt diagrams to the architectural context. Adaptation to print brings subtle changes, such as title pages, page numbers, and fully pointed text. The demands of a long-held calligraphic aesthetic ideal and the necessity to compete against traditional manuscripts within the educational marketplace combined to favor the use of lithography over typography in Arabic printed geometry.

The articles included in section I. $b$ all share the general goal of deepening our philosophical understanding of the diagrams of Euclid's geometry. In his paper, Marco Panza advances an account where geometric diagrams are fundamental to Euclid's plane geometry in fixing what the objects of the theory are. Specifically for Panza geometric diagrams perform two indispensable theoretical roles. First, they provide the identity conditions for the objects referred to in Euclid's propositions; second, they provide the basis via their concrete properties and relations for the attribution of certain properties and relations to these objects. After explicating this conception 
in general terms, Panza demonstrates what it amounts to vis-à-vis the Elements with a detailed and thorough analysis of the definitions and the first 12 propositions of book I.

A consequence of Panza's account is that Euclid's geometry is in a sense a constructive theory. It does not concern a pre-existing domain of objects, but instead objects brought into existence by the constructions of geometers. The issue of John Mumma's paper is the extent to which such an interpretation of Euclid can be given in formal terms. At the center of his discussion is a proof system he developed where Euclid's diagrams are formalized as part of the system's syntax. Mumma argues that his formalization is superior to others in accounting for the spatial character of the theory's constructions. It is not immediate, however, that the resulting picture of the theory qualifies as a constructive one. Mumma closes the article by pointing to the philosophical work that is needed for it to be understood as such.

A prima facie feature of the diagram based reasoning of Euclid's geometry is that perception is involved in a distinctive and essential way. But how is it so involved? The purpose of Annalisa Coliva's paper is to develop the philosophical concepts necessary to address the question. The key concept for Coliva, as the title of her paper indicates, is that of seeing-as. A segment joining opposite vertices of a square in a diagram can, for instance, be seen as a diagonal of the square, or the side of a triangle composing the square. Coliva articulates seeing-as as philosophically sharp notion, and argues that the ability is required to engage in diagram based geometric reasoning. She also offers some initial thoughts on how her analysis bears on the status of geometrical knowledge with respect to the a priori versus a posteriori and analytic versus synthetic distinctions.

In her paper, Sun-Joo Shin considers Euclid's diagrammatic arguments in order to examine commonly held views on the strengths and weaknesses of diagrams in comparison with symbolic/linguistic representations - namely the views that the latter are superior for giving proofs and the former are superior for brainstorming and discovery. Shin relates the first view to Locke and Berkeley's opposing accounts of the generality of Euclid's proofs, and argues that Berkeley's account offers a way for understanding diagrams as a legitimate means for general proofs. She proposes that a major reason behind the second view, aptly illustrated by Euclid's proofs, is that diagrammatic representations of individual mathematical objects are effective in generating the right associations and connections with what is already known.

Section I.c contains papers that explore how the diagrammatic method of Euclid's geometry influenced and/or was interpreted by thinkers in the early modern period. Though mathematics was pushing far beyond classical Greek geometry in this period, Euclid's Elements nevertheless maintained its position as a foundational text and thus formed part of the intellectual context in which philosophical or methodological reflections on mathematics were carried out.

Such reflections in Hume's Treatise form the subject of Graciela De Pierris's paper. In part II of Book I of the Treatise Hume asserts that perceivable yet indivisible minima compose geometric continua, but do so in a confused manner. Consequently, for Hume, geometry does not possess the exactness of arithmetic, which concerns clearly distinct discrete quantities. De Pierris argues that these doctrines follow in a principled way from an epistemological model in which phenomenologically given sensory 
images are fundamental. She also explains how Euclid's geometry for Hume, despite its relative inexactitude, qualifies as a demonstrative science in being based on simple, easily surveyable diagrams.

In her paper, Katherine Dunlop explores the role of diagrams in Newton's Principia by considering Newton's claim that the reasoning of the book is distinctively geometrical. She notes that Newton conceives geometry as the science of measurement, and argues that his characterization applies to the propositions of Section II, Book I. As other scholars have noted, these constitute a foundation in the sense that they show certain spatial quantities to be related as measures to nonspatial quantities, in particular, force and time. Dunlop takes Newton's point to be that the measures he supplies can be manipulated in the same way as elements of diagrams in classical geometry. In the case she examines in detail, Newton's proof of Kepler's area law, the principal measure of time is just the diagrammed trace of an inertial motion. Dunlop contends that Newton aims to share in geometry's certainty by resting determinations of equality and relative size (between nonspatial magnitudes) on relationships that are open to sensory inspection.

In the Critique of Pure Reason Kant often illustrates his claims with geometric proofs from Euclid's Elements. One thus might look to recent work on the diagrammatic character of these proofs to gain a better understanding of Kant's philosophy of geometry. In his article, Michael Friedman argues that such a project would be misdirected. He argues specifically that Kant's notion of a schema for geometrical concepts and his distinction between pure and empirical intuition precludes a diagrammatic interpretation of his theory of geometrical intuition. Such an interpretation would fail, for Friedman, to explain why Kant takes the constructions of Euclid's geometry to provide an a priori framework for empirical space. Friedman relates these points to Kant's theory of space, and the role of geometry and spatial intuition in the transcendental deduction of the categories.

Dominique Tournès's paper, the first of section II. $a$, serves nicely as bridge between the papers of part I and those of part II. In the article Tournès provides a survey of the changing role of diagrams in the history of differential equations from the seventeenth century-when there were still vestiges of the diagrammatic approach of Euclid's geometry — up until the time of Poincaré — when the influence of Euclid on mathematics was entirely absent. The article is a useful resource for those interested in understanding diagrams in mathematical subjects that are not ostensibly or exclusively geometrical in content.

The other contributions of section II. $a$ examine diagrams in the thought and work of two giant figures in the history and philosophy of modern mathematics.

Danniele Macbeth's proposes a new way of reading Frege's logical notation, as a sort of diagrammatic language within which to exhibit the contents of concepts. Taking Frege' s proof of theorem 133 in Part III of his 1879 treatise Begriffsschrift as an illustrative example, Macbeth aims to show just how a proof in Frege' s language (so read) can extend our knowledge despite being strictly deductive.

In his paper, Ivahn Smadja seeks to clarify how Hilbert understood the relative positions of diagrams and axioms in mathematics. An easy, and overly simplistic, characterization of Hilbert on the issue would be just to attribute to him the view the view Shin examines in her contribution-i.e. diagrams belong to the realm of heuris- 
tics and discovery while axiomatics belong to the separate realm of justification. That it would be overly simplistic is revealed by Smadja's study of the connections between the diagrams found in Minkowski's work in number theory and Hilbert's work in the foundations of geometry. Smadja specifically describes the links the former have to Hilbert's axiomatic investigations of the notion of a straight line, and Hilbert's concern with the conceptual compatibility of geometry and arithmetic.

The papers included in section II.c, the last group of papers, concern contemporary mathematics and linguistics. At the heart of Solomon Feferman's discussion are infinite diagrams for the proofs of central theorems in set theory, model theory and homological algebra. The diagrams are infinite in the sense that they depict infinitely iterated constructions. Feferman contends that they are more or less essential for understanding and accepting the proofs, and considers whether this serves as evidence against the so-called formalizability thesis: the idea that all mathematical proofs admit of formalization. He argues for the conclusion that it does not in the light of recent discussions of the formalizability thesis.

Sketch theory, a mathematical theory that treats the diagrams of category theory as mathematical objects, is the topic of Brice Halimi's paper. Halimi presents a technically sophisticated discussion of the theory in order to bring out its relevance to a variety of philosophical and foundational issues. These are: the capacity of a mathematical diagram (in category theory and elsewhere) to represent not just a static object but also the dynamics of a proof; the role and nature of diagrams within category theory; and the alternative ways category theory and set theory provide a semantics for mathematics.

A general point of many of the papers discussed so far is that diagrams can have important theoretical functions within mathematics. Arancha San Gines's paper provides evidence that the same is true in linguistics, in the most direct way possible. In it she presents a diagrammatic account of the role played by anaphoric pronouns in natural discourse. The account, specifically, is a diagrammatic system of representation for sentences containing such pronouns. After motivating and describing the system, she shows how it accounts for a variety of linguistic phenomena that have so far resisted uniform treatment.

John Mumma \& Marco Panza Stanford, Paris, May 2011

\section{References}

Allwein, G., \& Barwise, J. (Eds.). (1996). Logical reasoning with diagrams. New York: Oxford University Press.

Manders, K. (2008). The Euclidean diagram (1995). In P. Mancosu (Ed.), The philosophy of mathematical practice (pp. 80-133). New York: Oxford University Press.

Netz, R. (1999). The shaping of deduction in Greek mathematics. A study in cognitive history. Cambridge, USA: Cambridge U. P. 